\title{
Los proyectos de innovación como recurso formativo en el Máster del Profesorado
}

\author{
Isabel María Gómez Trigueros \\ Universidad de Alicante \\ Departamento de Didáctica General y Didácticas Específicas. \\ Isabel.gomez@ua.es
}

Fecha presentación: 30/7/2017 | Aceptación: 3/12/2017 |Publicación: 21/12/2017

\begin{abstract}
Resumen
Los cambios producidos en todos los ámbitos de la vida como consecuencia del desarrollo de la Sociedad de la Información y la Comunicación tiene su reflejo en la Educación Superior y en la necesaria adaptación tanto de los planes de estudio como de la formación del profesorado estas nuevas necesidades. En este sentido, desde el Espacio Europeo de Educación Superior se aboga por la formación en competencias así como por la implementación de metodologías activas con tecnología. En este estudio se propone la utilización de una metodología eminentemente activa, cooperativa y con tecnologías para la construcción de proyectos de innovación educativa como recurso para el desarrollo de buenas prácticas formativas. Se propone un análisis de tipo exploratorio descriptivo a través de un cuestionario escala Likert centrado en constatar las potencialidades de dicha estrategia de aula en la formación tecnológico-pedagógica docente para estudiantes de Máster del Profesorado. Los resultados presentan la percepción de la muestra respecto a la ausencia en la implementación de metodologías activas y a la incorrecta inclusión de las tecnologías en las prácticas de aula. Se concluye con una serie de consideraciones en la formación del profesorado.
\end{abstract}

Palabras clave: máster, innovación, Geografía, Historia.

\begin{abstract}
Resum
Els canvis produïts en tots els àmbits de la vida com a conseqüència del desenvolupament de la Societat de la Informació i la Comunicació té el seu reflex en l'Educació Superior i en la necessària adaptació tant dels plans d'estudi com de la formació del professorat aquestes noves necessitats. En aquest sentit, des de l'Espai Europeu d'Educació Superior s'advoca per la formació en competències així com per la implementació de metodologies actives amb tecnologia. En aquest estudi es proposa la utilització d'una metodologia eminentment activa, cooperativa i amb tecnologies per a la construcció de projectes d'innovació educativa com a recurs per al desenvolupament de bones pràctiques formatives. Es proposa una anàlisi de tipus exploratori descriptiu a través d'un qüestionari escala Likert centrat a constatar les potencialitats d'aquesta estratègia d'aula en la formació tecnològic-pedagògica docent per a estudiants de Màster del Professorat. Els resultats presenten la percepció de la mostra respecte a l'absència en la implementació de metodologies actives i a la incorrecta inclusió de les tecnologies en les pràctiques d'aula. Es conclou amb una sèrie de consideracions en la formació del professorat.
\end{abstract}

Paraules clau: màster, innovació, Geografia, Història.

\begin{abstract}
The changes produced in all areas of life as a result of the development of the Information Society and Communication is reflected in Higher Education and in the necessary adaptation of both curricula and teacher training these new needs. In this sense, the European Space of Higher Education advocates training in skills as well as the implementation of active methodologies with technology. This study proposes the use of an eminently active methodology, cooperative and with technologies for the construction of projects of educational innovation as a resource for the development of good training practices. A descriptive exploratory type analysis is proposed through a Likert scale questionnaire focused on verifying the potentialities of this classroom strategy in the technological-pedagogical teacher training for Master's students. The results show the perception of the sample regarding the absence in the implementation of active methodologies and the incorrect inclusion of the technologies in the classroom practices. It concludes with a series of considerations in teacher training.
\end{abstract}


Gómez Trigueros, Isabel María. “Los proyectos de innovación como recurso formativo en el Máster del Profesorado". @tic. revista d'innovació educativa. Número 19. Otoño (julio-diciembre 2017), pp. 22-29.

\section{Introducción y estado de la cuestión}

El uso generalizado de las tecnologías y los contextos interactivos de acceso a la información y a la comunicación han revolucionado los procesos de transferencia del conocimiento dando lugar a nuevos escenarios de formación, tipos de materiales y procesos de enseñanza y de aprendizaje innovadores. La innovación educativa implica la creación de teorías, de modelos, de técnicas y de métodos cada vez más rigurosos y valiosos así como su aplicación al proceso educativo desarrollado diariamente en las instituciones escolares.

La conceptualización de innovación educativa resulta compleja. Desde la dimensión educativa se afirma que es la acción permanente realizada mediante la investigación, para buscar nuevas soluciones a los problemas planteados en el ámbito educativo (Picardo, Escobar y Pacheco, 2005).

Del mismo modo, De la Torre (1994) considera que el término innovación se refiere a la introducción de nuevas dinámicas que alteran las ideas, las concepciones, las metas, los roles, los contenidos, la metodología, la organización espacial y temporal, los recursos o la evaluación en los ámbitos educativos.

Por su parte, Imbernón (1996) la define como la actitud y el proceso de indagación de nuevas ideas, propuestas y aportes cooperativos, para la solución de situaciones problemáticas docentes, que comporta un cambio en los contextos y en la práctica institucional de la educación. A tales propuestas, Cros y Adamczewski (1996) añaden que para que un proceso educativo sea considerado innovador debe dársele su espacio social, es decir, dimensionarlo en un contexto concreto y no limitarse a introducir un cambio en las dinámicas existentes. Esta idea enlaza con la necesidad de perdurabilidad en el tiempo de las acciones que cambian el contexto educativo. Así Carbonell (en Cañal de León, 2002) señala la necesidad de prolongar longitudinalmente las intervenciones de aula para que la renovación que propone la innovación sea patente y de sus frutos. En esta línea se encuentra también la aportación realizada por Sein-Echaluce, Fidalgo-Blanco y Alves que conciben innovar como "la aplicación de una idea que produce cambio planificado en procesos, servicios o productos que generan mejora en los objetivos formativos" (2017: 596-598).

Para Morin y Seurat (1998) se trataría de aplicar, en un contexto como es el aula y con un objetivo previamente diseñado, aquellas tecnologías creadas para otros ámbitos de la vida pero que incorporadas a la educación le confieren una auténtica novedad.

Es imprescindible añadir a tales definiciones aspectos como la importancia que presentan cada uno de los agentes involucrados. En este sentido, se plantea implicar a todo el sistema educativo, desde las Administraciones hasta al alumnado, proporcionando así aquellos elementos que converjan en un clima cambiante, de descubrimiento, reflexión, critico, deseable (Pascual, 1988).

Se debe poner de relieve que en todas estas aproximaciones al concepto de innovación se repite la idea de cambio respecto a las formas pedagógicodocentes existentes. Este cambio aparece ligado íntimamente a la incorporación de herramientas tecnológicas. No se debe obviar que sigue siendo común relacionar innovación educativa con la instalación de ordenadores y otros recursos electrónicos en las aulas, confundiendo la modernización de los centros de enseñanza con los procesos de mejora y renovación educativa. Queda constatado que tal fenómeno no supone por si solo un verdadero cambio pedagógico, ya que el uso que se viene haciendo de la tecnología instalada continua siendo el de mero emisor de contenidos a modo de la pizarra tradicional (Meneses, 2007). En particular, se presta especial atención a Internet y a la conexión de los centros que surge como imprescindible para lograr la tal deseada innovación educativa al vincularla con comunicación y transmisión. Esta idea de transmitir aquellos logros educativos se opone en sí a aquella típica actitud docente consistente en guardar fórmulas magistrales para la enseñanza. Se vislumbra así, en relación a los procesos innovadores la conciencia de generar nuevos conocimientos que confluyen en una mejora de los procesos docentes de toda la comunidad educativa. En este contexto la innovación educativa se contempla como sinónimo de renovación pedagógica y se puede definir como un conjunto de estrategias bien estructuradas que tienen como objetivo prioritario modificar las prácticas educativas tradicionales (Cañal de León, 2005). Supone un proceso que se prolonga en el tiempo y que converge en un proceso de reforma de la metodología, la pedagogía, la organización de los contenidos y el uso de herramientas desde una dimensión diferente a la utilizada hasta el momento.

En relación a los paradigmas que acogen los diversos estudios sobre innovación educativa, se diferencian (resumiendo enormemente la ingente cantidad de trabajos y estudios realizados) dos grandes líneas: de un lado el paradigma interpretativo también considerado técnico-academicista que incide en los supuestos conceptuales y los referentes intelectuales como pensamiento implícito en las prácticas de los docentes (Zoppi, 1995); de otro lado el reflexivo-crítico centrado en analizar los diferentes recursos del aula y la actitud necesaria del docente como elemento activo del proceso de cambio en la práctica docente (Lucarelli, 1996).

Si bien es cierto, como señala Cañal de León que la gran mayoría de propuestas didácticas adolecen de "fundamentación o referentes teóricos" (2005: 15) sí que se descubren, en las intervenciones más recientes (Cózar, Zagalaz y Sáez, 2015; Blanch, Betancort y Martínez, 2016; Ortega y Gómez, 2017; Gómez y Ruiz), un mayor interés por sustentar conceptualmente las prácticas educativas innovadoras, dotándolas de un sustrato teórico que permita validar los resultados alcanzados.

Para este trabajo se ha considerado la innovación docente como aquellas propuestas e intervenciones generadas por docentes para el aula en las que se procede a la ruptura respecto a las prácticas vigentes consolidadas, orientadas básicamente a la adquisición de contenidos curriculares, y que atienden tanto a los intereses y motivaciones de los docentes que las diseñan y lideran como a los de su alumnado con el objetivo central de adquirir conocimientos y competencias, clave imprescindibles para el desarrollo de una ciudadanía crítica (Delors, 1996).

\section{Método}

\subsection{Contexto de la intervención y participantes}

La intervención analizada en este estudio se ubica en el contexto de la Facultad de Educación de Alicante a lo largo del curso académico (2016-2017), en las 
Gómez Trigueros, Isabel María. “Los proyectos de innovación como recurso formativo en el Máster del Profesorado". @tic. revista d'innovació educativa. Número 19. Otoño (julio-diciembre 2017), pp. 22-29.

asignaturas de "Innovación e Investigación en Didáctica de la Geografía” e "Innovación e Investigación en Didáctica de la Historia, Historia del Arte y Ciudadanía" del Máster de Profesorado de Educación Secundaria. La muestra del estudio está formado por 92 participantes cuyo valor representa el $100 \%$ de la población total de los estudiantes de Máster de dicha universidad, y por tanto, se sitúa dentro de los parámetros representativos en este tipo de herramienta que es el cuestionario (Kaplowitz, Hakdlock y Levine, 2004).

Su distribución por cursos y género se concreta en la Figura 1:

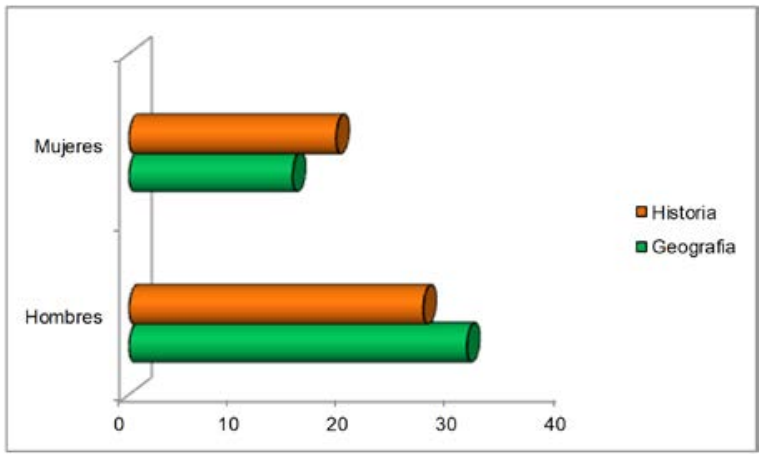

Figura 1. Características de género de la muestra.

La muestra es similar y representativa de otros cursos académicos anteriores con un total de $37 \%$ de mujeres frente a un $63 \%$ de hombres. En referencia al rango de edad, no existen diferencias significativas pues el $98,3 \%$ se encuentran entre los 21 y los 22 años y el $100 \%$ finalizó sus estudios de Grado de Geografía o de Historia durante el año 2015.

\subsection{Diseño de la investigación y metodología}

La investigación que se presenta es descriptiva y se ha utilizado un modelo mixto de tipo exploratorio mediante encuesta (Johnson, 2004) cuyo objetivo central ha sido mostrar el estado de la cuestión sobre los proyectos de innovación docente y tener una visión amplia del fenómeno analizado. Dicho enfoque se ha organizado en torno a dos fases diferenciadas: de un lado la fase inicial de recogida de datos cualitativos y cuantitativos; de otro, la segunda fase en la que se ha construido una base de datos en la que se han integrado los dos tipos de resultados para el desarrollo de análisis mixtos (García, 2011).

En la fase inicial se propone un cuestionario escala Likert de respuesta online, distribuido por Campus Virtual de la Universidad de Alicante. El alumnado participante debe responder a las preguntas planteadas previamente a la intervención didáctica en el aula. Posteriormente, finalizada la acción formativa, se vuelve a distribuir dicho cuestionario para constatar los cambios producidos respecto a los objetivos iniciales planteados en el estudio.

En la segunda fase, y con la finalidad de plantear la integración de nuevos contenidos con la práctica docente, se ha propuesto el trabajo cooperativo y colaborativo a través de pequeños grupos de estudiantes (no más de cuatro por equipo) para la elaboración de distintos proyectos de innovación docente en Ciencias Sociales. Los trabajos se deben circunscribir al nivel educativo de Educación Secundaria de manera que los estudiantes participantes puedan poner en práctica aquellos conocimientos previos adquiridos a lo largo del Grado de Geografía y del Grado de Historia respectivamente. Asimismo, todos los proyectos han de contener las directrices señaladas por la Conselleria d'Educació en materia de innovación docente y que aparecen recogidas en la normativa (DOCV núm. $7838 / 27 / 07 / 2016^{1}$ ). Como entrega del trabajo, los grupos de estudiantes deben presentar y defender su proyecto, incidiendo en los contenidos a desarrollar, competencias a adquirir y objetivos de su propuesta didáctica innovadora.

\section{Instrumento}

La medición de las variables analizadas se realiza mediante un cuestionario mixto, validado por diversos expertos de universidades españolas (Universidad de Burgos, Universidad Miguel Hernández de Elche, Universidad de Alicante) así como de áreas diversas (Ciencias Sociales, Sociología, Estadística).

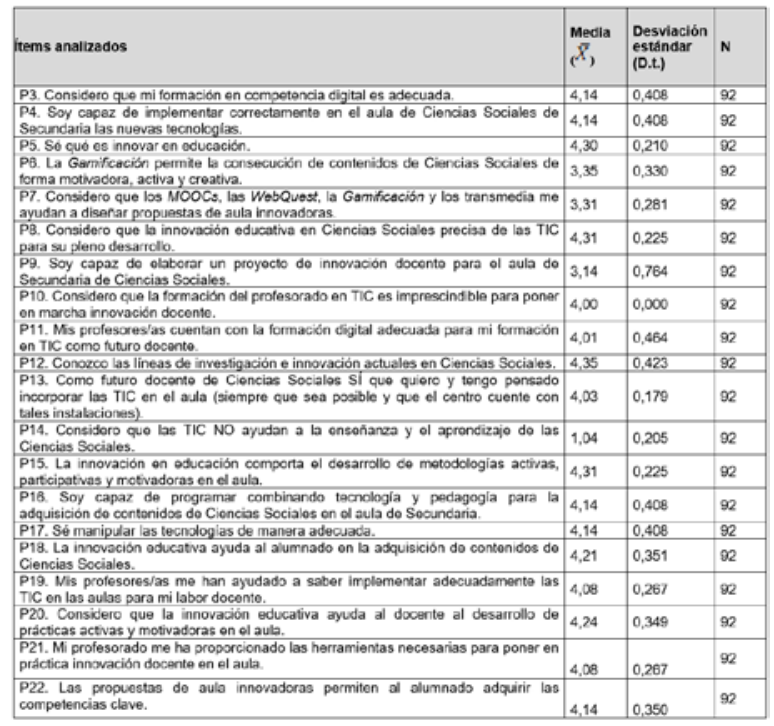

Figura 2. Datos estadísticos descriptivos, media, desviación estándar del cuestionario de análisis.

Los elementos que configuran tal instrumento se organizan en dos secciones diferenciadas: de un lado, los rasgos sociodemográficos como género y edad y, de otro, los componentes relacionados con la percepción de los participantes respecto a su formación a través de prácticas innovadoras, activas, colaborativas y motivadoras; la transferencia que reciben de su profesorado respecto de las tecnologías como herramientas docentes así como la adecuada implementación de las mismas en la enseñanza y el aprendizaje (E-A) de las Ciencias Sociales. En este sentido, se analiza la competencia digital de la muestra; la destreza en la manipulación de las tecnologías y su capacidad de implementación didáctico-pedagógica en un proyecto de innovación docente para el aula de Geografía, Historia y/o Historia del Arte de Enseñanza Secundaria (Figura 2). La escala utilizada es tipo Likert, de cinco opciones de respuesta que oscilan entre Muy en

\footnotetext{
1 Diari Oficial de la Comunidad Valenciana [DOCV] (2016). Recuperado el 14 de abril de 2017, de:

http://www.docv.gva.es/datos/2016/07/27/pdf/2016_6018.p df
} 
Gómez Trigueros, Isabel María. “Los proyectos de innovación como recurso formativo en el Máster del Profesorado”. @tic. revista d'innovació educativa. Número 19. Otoño (julio-diciembre 2017), pp. 22-29.

desacuerdo (valor 1) a Totalmente de acuerdo (valor 5). Del mismo modo, la evaluación de las exposiciones orales (segunda fase de la investigación) se realiza siguiendo una plantilla de formato rúbrica (Figura 3) confeccionada por el área de Ciencias Sociales del departamento de Didáctica General y Didácticas Específicas de la Facultad de Educación, validado por expertos en didáctica del Grupo de Innovación Docente UBU (GID UBU en Didáctica de las Ciencias Sociales, de las Lenguas y de las Literaturas en la formación inicial del profesorado de Educación Infantil y Educación Primaria).

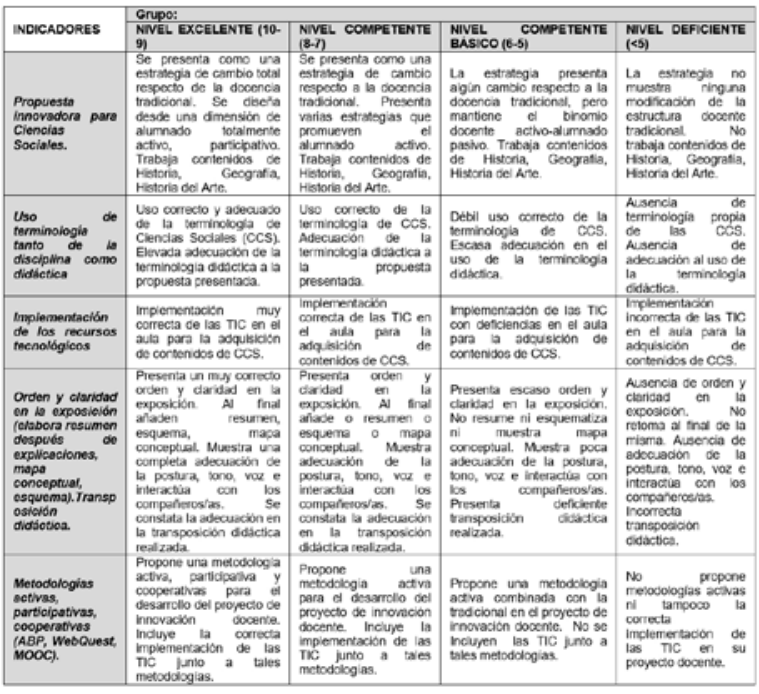

Figura 3. Rubrica para evaluación de las propuestas de innovación docente de la muestra participante.

La valoración de las distintas exposiciones se ha desarrollado siguiendo la estrategia de evaluación entre iguales $^{2}$ (Falchikov y Goldfinch, 2000; Sivan, 2000) por considerarla como la manera más adecuada de evaluar las distintas propuestas.

\section{Resultados}

\subsection{Fiabilidad del instrumento}

Para valorar la fiabilidad y la consistencia interna del instrumento utilizado en este trabajo (cuestionario) se ha hallado el estadístico Alfa de Cronbach $(\alpha)$ eliminando del análisis los ítems cualitativos (sexo y edad de la muestra), arrojando un resultado de 0,951 ( $\alpha=.951)$ indicativo de una alta fiabilidad y consistencias (Bisquerra et al, 2004; Huh, Delorme y Reid, 2006). Asimismo, se han calculado aquellos descriptivos reveladores como la media $\left({ }^{\bar{X}}\right)$ y la desviación típica(D.t.).

\footnotetext{
2 Según apuntan diferentes estudios planteados por Soledad, Rodríguez y Gómez (2012) existen resultados que constatan el valor de la evaluación entre iguales en cualquier disciplina así como en ambientes donde se implementen metodologías activas y colaborativas entre el alumnado. En este sentido, se considera ideal para el trabajo con proyectos de innovación docente del Máster de Profesorado de Secundaria proponer este tipo de recurso como evaluación de las propuestas diseñadas.

3 Método de extracción: análisis de componentes principales. Método de rotación: Varimax con normalización Kaiser
}

\begin{tabular}{|c|c|c|c|}
\hline \multicolumn{4}{|c|}{ Matriz de componente rotado ${ }^{3}$} \\
\hline \multirow{2}{*}{ Ítems } & \multicolumn{3}{|c|}{ Componente } \\
\hline & 1 & 2 & 3 \\
\hline B3 & 0,891 & & \\
\hline B4 & 0,891 & & \\
\hline B5 & & 0,915 & \\
\hline B6 & & 0,844 & \\
\hline B7 & & 0,855 & \\
\hline B8 & & 0,807 & \\
\hline B9 & & 0,918 & \\
\hline B11 & & & 0,655 \\
\hline B12 & & 0,804 & \\
\hline B13 & 0,764 & & \\
\hline B14 & 0,821 & & \\
\hline B15 & & 0,856 & \\
\hline B16 & 0,891 & & \\
\hline B17 & 0,891 & & \\
\hline B18 & & 0,875 & \\
\hline B19 & & & 0,795 \\
\hline B20 & & 0,856 & \\
\hline B21 & & & 0,795 \\
\hline B22 & & 0,856 & \\
\hline
\end{tabular}

Figura 4. Contribución de los ítems a los componentes principales extraídos con rotación VARIMAX.

El programa empleado para extraer tales estadísticos ha sido el SPSS en su versión 23.

Del mismo modo, se ha realizado el análisis factorial con rotación VARIMAX que mostró tres factores o componentes principales de reducción de datos el primer componente relacionado con la percepción de los estudiantes de Máster sobre su formación en competencia digital; el segundo componente relativo a los conocimientos de la muestra en relación a la innovación docente y a las TIC, y a su importancia en el proceso de adquisición de contenidos y competencias clave que explican el $67 \%$ de la varianza y el tercero referente a la capacitación de los/las docentes de Máster del Profesorado en tecnologías y en la enseñanza para su correcta inclusión en las aulas. Asimismo, se comprueba una elevada covarianza entre los factores así como una elevada contribución de los distintos ítems a la dimensión o componente que conforman (Figura 4).

Respecto a los datos cualitativos obtenidos a través de la rúbrica de evaluación de las prácticas realizadas se observa una clara coincidencia entre las respuestas del cuestionario y las propuestas presentadas. En este sentido, destacan los proyectos innovadores calificados como sobresaliente $(60 \%)$ seguido por los que superan la calificación de 7-8 (30\%) de la evaluación entre iguales. Del mismo modo, no se ha producido ninguna calificación inferior a 5 indicativo de la plena comprensión del alumnado participante sobre las directrices y los elementos que debe contener un proyecto docente innovador (Figura 5). 
Gómez Trigueros, Isabel María. “Los proyectos de innovación como recurso formativo en el Máster del Profesorado". @tic. revista d'innovació educativa. Número 19. Otoño (julio-diciembre 2017), pp. 22-29.

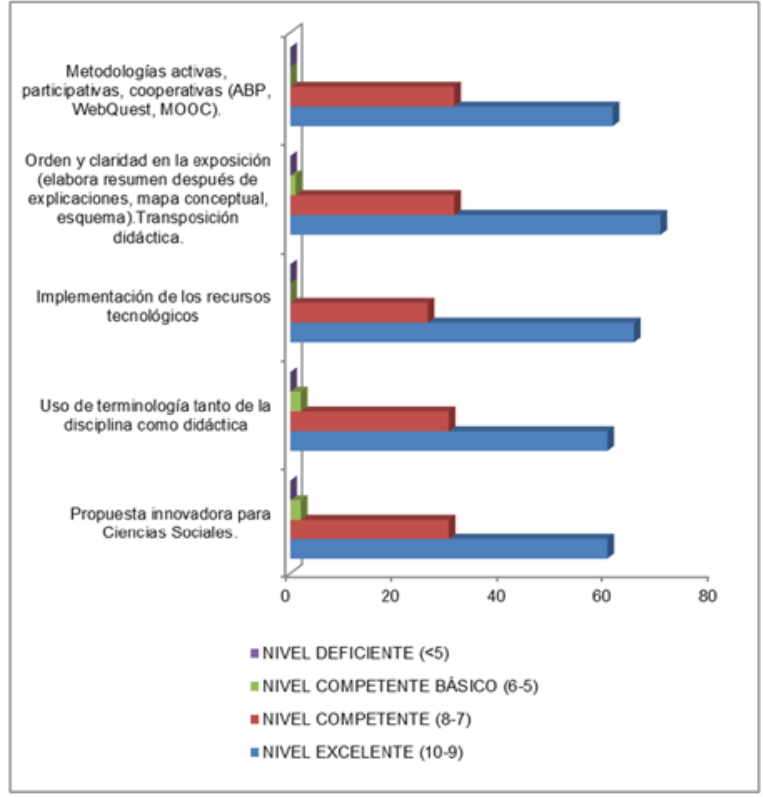

Figura 5. Resultados de la evaluación entre iguales de la práctica de aula sobre exposiciones de proyectos de innovación docente de la muestra.

\subsection{Análisis descriptivo los componentes principales del estudio}

El análisis descriptivo muestra los resultados de la media $\left({ }^{\bar{X}}\right)$ y de la desviación típica (D.t.) de los ítems del cuestionario en base a la escala Likert empleada (Figura 4). Los valores en términos generales indican que la muestra participante, futuros/as docentes de Ciencias Sociales, tienen una actitud positiva hacia la utilización de las tecnologías en el proceso de E-A como se constata en los resultados de los ítems 10 y 13 (valor ${ }^{\bar{X}}$ entre 4,00-4,03; D.t. <0,200).

En este sentido, las medias estadísticas muestran valores muy positivos señalando que la mayor parte de los estudiantes participantes responden con la opción "De acuerdo" y "Totalmente de acuerdo" $(\bar{X} \geq 4,00)$ respecto a la importancia de las TIC en Secundaria como herramientas favorecedoras para el aprendizaje de Ciencias Sociales. Igualmente, se comprueba una reducida desviación típica inferior a 0,6 (D.t. <0,200) incidiendo en la convergencia de respuestas altamente positivas de la mayoría de la muestra analizada.

Del mismo modo, el ítem 14 confirma esta percepción altamente positiva hacia las tecnologías como herramientas para el aula con un elevado número de respuestas "Totalmente en desacuerdo" ante la cuestión planteada $(\bar{X}=1,04)$. Como en el caso anterior, la opción de respuesta es coincidente en la mayoría de los/las participantes en el estudio (D.t. $=0,205)$.

Si se valora la percepción de la muestra respecto a su propia capacitación tecnológica se observa que los estudiantes consideran tener una buena formación digital. Así, en referencia a su formación en la manipulación de los recursos electrónicos, que aparece recogida en los ítems 3 y 17, el análisis descriptivo demuestra que los estudiantes la cuantifican como muy positiva con una aproximación al valor 5 de respuesta $\left({ }^{\bar{X}}=4,14\right)$ relativo a la opción "Totalmente de acuerdo". Algo similar ocurre cuando se les pregunta sobre su formación pedagógica en cuanto a su capacidad para incorporar adecuadamente las TIC en el aula (ítems 4 y 16) con resultados de respuesta similares $(\bar{X}=4,14)$ como se constata en la Figura 4 . En este sentido, se quiere destacar la coincidencia en las respuestas de los/las participantes sobre la su capacitación pedagógico-digital, con una desviación típica inferior en todos los casos a 0,6 (D.t. $=0,408)$.

En referencia al segundo de los componentes extraídos (Figura 4), relacionado con los conocimientos de la muestra sobre innovación docente y su importancia para la adquisición de contenidos curriculares así como de competencias clave, se debe indicar que aunque la muestra analizada estima que cuenta con un conocimiento amplio del concepto "innovación educativa" (ítem 5) y un gran número de los/las estudiantes afirma ser conocedora de las líneas actuales de investigación e innovación docente para el aula de Ciencias Sociales (ítem 12) no ocurre igual cuando se les pregunta sobre algunas de las innovaciones tecnológicas y pedagógicas actuales (ítems 6 y 7 ). En este sentido, los descriptivos del ítem 5 se aproximan al valor 5 $\left({ }^{\bar{X}} \geq 4,14\right)$ de respuesta ("Totalmente de acuerdo") en la mayoría de los casos (D.t. $<0,600$ ) mientras que los resultados obtenidos en las cuestiones 6 y 7 se decantan por la opción "Ni de acuerdo ni en desacuerdo" $\left({ }^{\bar{X}} \geq 3,35\right.$ y 3,31 respectivamente) indicativo de la falta de formación sobre tales metodologías y herramientas innovadoras.

Otro aspecto que recoge el instrumento utilizado hace referencia a la relación entre TIC e innovación docente plasmada en las respuestas del ítem 8 con una media próxima al valor 5 de respuesta $\left({ }^{\bar{X}}=4,31\right)$ equivalente a "Totalmente de acuerdo". Dichos resultados confirman la percepción del alumnado participante sobre la íntima relación entre innovación y tecnologías. De igual forma, la desviación típica inferior al valor 6 (D.t. $=0,225)$ supone que dicha creencia es generalizada entre los estudiantes de Máster de Profesorado de Secundaria. Algo similar sucede cuando se les consulta sobre la relación existente entre innovación docente y la implementación de metodologías activas en el aula (ítem 15) y donde la mayoría de respuestas obtenidos (D.t. $=0,225)$ ha sido "Totalmente de acuerdo" $(\bar{X}=4,31)$ indicativo de representación que los/las futuros/as docentes tienen respecto a tal binomio. En esta misma línea se encuentran los resultados de los ítems 18 y 20 sobre la importancia de la innovación para la adquisición de conocimientos por parte de los estudiantes como para la propia formación inicial del profesorado. Así, los resultados del análisis descriptivo arrojan un valor de media superior a $4,00\left({ }^{\bar{X}}\right.$ de 4,21 y 4,240

respectivamente y una desviación típica inferior a 0,6 (D.t. de 0,351 y 0,349 para cada uno de los ítems) indicativo de la importancia que la muestra otorga a la innovación no sólo para la adquisición de conocimientos nuevos sino también para la correcta formación de los docentes.

Tales datos contrastan con las respuestas del ítem 9 ( ${ }^{\bar{X}}=3,14$; D.t. $\left.=0,764\right)$ relativa a la concepción del alumnado en relación a su capacidad para elaborar proyectos de innovación docente para el aula de Ciencias Sociales. Estos resultados son indicativos de la carente formación del futuro profesorado en materia de innovación, percibido por los propios estudiantes, futuros/as docentes de Secundaria. 
Gómez Trigueros, Isabel María. “Los proyectos de innovación como recurso formativo en el Máster del Profesorado". @tic. revista d'innovació educativa. Número 19. Otoño (julio-diciembre 2017), pp. 22-29.

Por lo que respecta al tercer componente analizado, relativo a la capacitación del profesorado de Máster sobre tecnologías e innovación, recogido en las cuestiones 11, 19 y 21 se puede observar que la mayoría del alumnado percibe que el profesorado de Máster cuenta con la preparación tecnológica, metodológica y pedagógica adecuada con un mayor número de respuesta de la opción "De acuerdo" $\left(\bar{X}_{\geq 4,00 ;}\right.$ D.t.<0,6). Además, se constata que consideran adecuadas las herramientas y nociones proporcionadas por los docentes en su formación inicial para su futura labor como enseñantes (ítem 19 y 21) significativo de que los problemas que presentan para la elaboración de proyectos de innovación docente no se debe a la atención recibida en sus clases de Máster sino que podría deberse a las dificultades encontradas para proponer una correcta implementación de las TIC en el aula.

\section{Discusión y conclusiones}

A la vista de los datos obtenidos y ante la proliferación de la implementación de nuevas metodologías activas así como a la preocupación de los/las docentes por la correcta implementación de las TIC en la educación superior, es necesario el replanteamiento de los modelos y las estrategias de E-A en la formación inicial del profesorado.

Aunque se ha indicado la existencia de estudios previos que han propuesto el trabajo de aula desde la dimensión de la incorporación herramientas y prácticas docentes relacionados con el concepto de innovación estas se han limitado, como señalan algunos autores (Morin y Seurat, 1998; Fullan, 2002) a la valoración de los cambios relacionados con la incorporación de las últimas tecnologías o a la simple introducción de algunas estrategias didácticas de aula de no más allá de tres o cuatro sesiones. Otros trabajos inciden en innovación docente sólo desde una dimensión organizativa de los agentes implicados en el proceso educativo (Havelock y Huberman, 1980; Curry, 1992; Havelock y Zlotolow, 1995) lo que requiere cambios tanto en el sistema de EA como la posterior instauración de los mismos y estabilización de dichas modificaciones en el funcionamiento natural de los centros (Salinas, 2004).

Por el contrario, son prácticamente nulos los trabajos sobre la percepción y cualificación del profesorado en formación, en innovación docente, centrándose los existentes en el análisis de las tecnologías y en la formación digital de los estudiantes sin incidir en la capacidad de los/las futuros/as docentes para la correcta inclusión de las TIC. Del mismo modo, los escasos trabajos encontrados prescinden de evaluar el uso de herramientas tecnológicas para el desarrollo de metodologías activas y participativas para el aula.

En este sentido, conocedores de la complejidad para la evaluación de actitudes o percepciones y dada la necesidad de contar con datos fiables para la valoración de intervenciones innovadoras semejantes a la que se ha propuesta en este estudio, se considera relevante el cuestionario y la rúbrica mostrada. Tales instrumentos han permitido conocer la funcionalidad e importancias de desarrollar propuestas docentes innovadoras en las clases de Máster del Profesorado de Secundaria, con el objetivo de formar profesionales mejor formados y aptos para afrontar los rápidos cambios que subyacen con la generalización de las tecnologías en la actual Sociedad de la Información y la Comunicación (Gómez, 2010). Tras el trabajo realizado se puede constatar que el alumnado participante valora muy positivamente las TIC y su incorporación en las aulas para la adquisición de contenidos de Ciencias Sociales. Además, los resultados obtenidos coinciden en señalar la riqueza formativa que ofrece el aprendizaje recibido en relación a la correcta inclusión de las tecnologías en el aula para su propia formación como futuros/as docentes, conclusión que coincide con la de otros estudios previos llevados a cabo por otros/as investigadores/as (Cardona, Fandiño, Jairo, 2014; Roig, Mengual, Sterrantino y Quinto, 2015). Los datos analizados confirman que los estudiantes no perciben problemas en el uso que ellos/as hacen de las tecnologías desde la dimensión manipulativa aunque sí concluyen en la existencia de dificultades para llevar a cabo una correcta utilización pedagógica de las mismas. Tales resultados nos llevan a corroborar la urgente necesidad de promover cambios sustanciales en los planes de estudio para la formación docente que hagan hincapié en la implementación didáctica de las herramientas tecnológicas.

En cuanto a la valoración que hacen los estudiantes sobre los proyectos de innovación docente, se puede concluir que los consideran instrumentos muy positivos para la E-A. En este sentido, se cuantifica un amplio consenso sobre la funcionalidad, en la formación inicial del profesorado, de la confección propuestas desde la innovación educativa ya que contribuyen a generar pensamientos flexibles, activos, participativos y creativos sobre la interconexión de conocimientos y recursos. Asimismo, su utilización facilita la visión útil de las metodologías activas y novedosas para la comprensión de la Geografía y la Historia, fomentando una mejora en la adquisición de contenidos curriculares de ambas materias que, de otro modo, podrían resultar difíciles de asimilar para el aula de Educación Secundaria. Como ya se ha señalado, la puesta en práctica de intervenciones de aula innovadoras conlleva cambios sustanciales en las estrategias y metodologías educativas favoreciendo la adquisición de contenidos y de competencias clave que ayudarán a la mejora del rendimiento del alumnado. Cabe señalar que dicha percepción eminentemente positiva hacia la innovación docente concuerdan con los de otros estudios que consideran necesario implementar nuevas estrategias metodológicas y tecnológicas en la docencia (Báez, 1994; Salinas, 2004; Gómez y Ruiz, 2017).

Por último, se debe destacar el reconocimiento y la positiva valoración que muestran los resultados obtenidos sobre la adecuación del profesorado de Máster en competencia digital. Los datos inciden en la importancia que los/las futuros/as docentes otorgan a la formación tecnológica de sus docentes así como a la capacidad de estos para proporcionar correctas pautas de inclusión de TIC y metodología innovadora.

Con respecto a las limitaciones del estudio realizado, se debe señalar que al tratase de un estudio exploratorio inicial de un solo año académico quedan pendientes los datos que se puedan extraer de otros cursos en el futuro. Del mismo modo, sería interesante incorporar resultados relativos a otras universidades españolas para poder llevar a cabo un estudio comparativo más complejo y extenso que permitiera obtener conclusiones definitivas sobre la innovación y la percepción general de los/las profesores en formación. Tales datos serían eminentemente útiles para promover e incorporar ciertas modificaciones en los actuales planes de estudio universitario.

En conclusión, la formación inicial del profesorado debe 
Gómez Trigueros, Isabel María. “Los proyectos de innovación como recurso formativo en el Máster del Profesorado". @tic. revista d'innovació educativa. Número 19. Otoño (julio-diciembre 2017), pp. 22-29.

tener en cuenta las propuestas didácticas innovadoras y la correcta inclusión de la tecnología como estrategia metodológica activa de intervención en el aula. A través de ellas, el alumnado adquiere nuevos contenidos y competencias clave que le ayudarán a desarrollar habilidades cognitivas de alto orden (Nisbet, 1991). Del mismo modo, al tratarse de propuestas de trabajo grupal, contribuyen a promover valores entre estudiantes y docentes tales como confianza, paciencia, paciencia, respeto, aceptación de los otros/as, movilidad en la diversidad y aceptación de nuevos roles, entre otros (Torres, 1996). Ayuda a conseguir un diálogo constructivo, crítico y permanente (Gómez y Ruiz, 2017) entre iguales y entre ellos/as y el profesorado. Asimismo, este tipo de experiencias dota a los/las futuros/as docentes de las competencias en el uso manipulativo y didáctico de las herramientas digitales ya mencionadas para el desarrollo de sus intervenciones en el aula y al diseño de estrategias eminentemente novedosas que permiten alcanzar el Conocimiento Base del Docente que "implica el dominio de las teorías y de las estrategias que generan más conocimiento, tanto desde un punto de vista pedagógico como metodológico y técnico" (Cabero, Marín y Castaño, 2015; 17).

\section{Bibliografía}

Báez de la Fe, Bernado (1994). "El movimiento de escuelas eficaces: Implicación para la innovación educativa". Revista Iberoamericana de Educación, 4,pp.93-116.

Blanch Marcos de León, Montserrat; Betancort Santos, Sonia y Martínez Avidad, Mayra (2016). "El Videoblog en el Aula de Lengua y Literatura de Secundaria. Una propuesta práctica". REICE. Revista Iberoamericana sobre Calidad, Eficacia y Cambio en Educación, 14(3),pp.33-39. Doi: 10.15366/reice2016.14.3.002

Bisquerra, R. (Coord.) (2004). Metodología de la investigación educativa. Madrid: La Muralla.

Cabero Almenara, Julio; Marín Díaz, Verónica y Castaño Garrido, Carlos (2015). "Validación de la aplicación del modelo TPACK para la formación del profesorado en TIC". @tic: revista d'innovació educativa, 14, 13-22.DOI: 10.7203/attic.14.4001.

Cañal de León, Pedro (2005). La innovación educativa. Madrid:Akal.

Cardona, Aurora y Fandiño, Yamith; Galindo, Jairo (2014). "Formación docente: creencias, actitudes y competencias para el uso de TIC". Lenguaje, 42(1), pp.173-208.

Cózar Gutiérrez, Ramón; Zagalaz, Juan y Sáez López, José Manuel (2015). "Creando contenidos curriculares digitales en Ciencias Sociales para Educación Primaria. Una experiencia TPACK para futuros docentes". Educatio Siglo XXI, 3 (3), pp.147-168.

Cros, Françoise y Adamczewski, Georges (1996). L' innovation en éducation el enformation. París: De Bóeck Université.

Curry, Barbara (1992). Instituting enduring innovations: achieving continuity of change in higher education. Washington, DC: ASHE-ERIC
Higher Education Reports.

De la Torre, Saturnino (1994). Innovación curricular. Procesos, estrategias y evaluación. Madrid: Editorial Dykinson.

Delors, Jacques (1996.). "Los cuatro pilares de la educación". En La educación encierra un tesoro. Informe a la UNESCO de la Comisión internacional sobre la educación para el siglo XXI (91-103), Madrid, España: Santillana/UNESCO.

Diari Oficial de la Comunidad Valenciana [DOCV] (2016). Recuperado el 14 de abril de 2017, de: http://www.docv.gva.es/datos/2016/07/27/pdf/2016 6018.pdf.

Falchikov, Nancy y Goldfinch, Judy (2000). "Student peer assessment in higher education: a metaanalysis comparing peer and teacher marks". Review of Educational Research, 70(3), pp.287322.

DOI:http://dx.doi.org/10.3102/00346543070003287

Field, M. (1994). "Assessing Interdisciplinary Learning". New Directions for Teaching and Learning, 58, pp.69-84.DOI: http://dx.doi.org/10.1002/tl.37219945806

Fullan, Michael (2002). Liderar en una cultura de cambio. Barcelona: Octaedro.

García Pérez, Alfonso (2011). Técnicas actuales de estadística aplicada. Madrid: UNED.

Gómez Trigueros, Isabel María (2010). "Análisis del paisaje físico y humano de la provincia de Alicante: Google Earth como herramienta docente en las clases de Geografía". GeoGraphos. Revista Digital para Estudiantes de Geografía y Ciencias Sociales, 1(1) 1-26. http://dx.doi.org/10.14198/GEOGRA2010.1.01

Gómez Trigueros, Isabel María y Ruiz Bañuls, Mónica (2017). "Análisis sobre nuevas metodologías activas basadas en el ABP y en la Gamificación en los estudios de Máster del Profesorado en Educación Secundaria". En: Roig-Vila, Rosabel (coord.). REDES-INNOVAESTIC 2017 (47-48), Alicante: Instituto de Ciencias de la Educación (ICE) de la Universidad de Alicante.

Havelock , Ronald y Huberman, Michael (1980). Innovación y problemas de la educación: teoría y realidad en los países en desarrollo. Ginebra: UNESCO.

Havelock, Ronald y Zlotolw Steve. (1995). The change agent's guide. Englewood Cliffs (NJ): Educational Technology Publications.

Huh, Jisu; Delorme, Denise; Reid, Leonard (2006). "Perceived ThirdPerson Effects and Consumer Attitudes on Prevetting and Banning DTC Advertising". Journal of Consumer Affairs, 40, 1, pp.90-116. DOI: http://doi.org/dpj596.

Ibarra Sáiz, María Soledad; Rodríguez Gómez, Gregorio y Gómez Ruiz, Miguel Ángel (2012). "La evaluación entre iguales: beneficios y estrategias para su práctica en la Universidad". Revista de Educación, 359. DOI: 10-4438/1988-592X-RE2010-359-092 
Gómez Trigueros, Isabel María. “Los proyectos de innovación como recurso formativo en el Máster del Profesorado”. @tic. revista d'innovació educativa. Número 19. Otoño (julio-diciembre 2017), pp. 22-29.

Imbernon, Francisco (1996). En busca del discurso educativo: la escuela, la innovación educativa, el curiculum el maestro y su formación. Buenos Aires: Magisterio del Río de la Plata.

Johnson, Burke y Onwuegbuzie, Anthony (2004). "Mixed methods research: A research paradigm whose time has come". Educational Researcher, 33(7), pp.14-26. DOI: http://dx.doi.org/10.3102/0013189X033007014.

Kaplowitz, Michael; Hadlock, Timoty y Levine, Ralph (2004). "A comparison of Weand mail survey response rates". Public Opinion Quarterly, 68, 94101.

Lucarelli, Elisa (1996) La construcción de la articulación teoríapráctica en las cátedras universitarias: búsquedas y avances. Instituto de Ciencias de la Educación- Facultad de Filosofia y Letras- Universidad de Buenos Aires.

Meneses Benítez, Gerardo (2007). "Universidad: NTIC, interacción y aprendizaje". Pixel-Bit. Revista de Medios y Educación, 29, pp. 45-58. http://dx.doi.org/10.21556/edutec.2006.20.518

Morin, Jacques y Seurat, Richard (1998). Gestión de los recursos tecnológicos. Madrid: Cotec.

Nisbet, John (1991). "Investigación reciente sobre estrategias de aprendizaje y pensamiento en la enseñanza". En Monereo, Carles (Comp.), Enseñar a pensar a través del curriculum escolar. (Ponencias de las II Jornadas de Estudio sobre Estrategias de Aprendizaje). Barcelona: Casals.

Ortega Sánchez, Delfín y Gómez Trigueros, Isabel María (2017). "Las WebQuests y los MOOCs en la enseñanza de las Ciencias Sociales y la formación del profesorado de Educación Primaria". Revista. Electrónica Interuniversitaria de Formación del Profesorado, 20(2), pp.205-220. Doi:10.6018/reifop.20.1.258551.

Pascual, Robert (1988). La gestión educativa ante la innovación y el cambio. Madrid: Narcea.

Picardo Joao, Oscar; Escobar Baños, Juan Carlos y Pacheco Cardoza, Rolando Valmore (2005). Diccionario Enciclopédico de Ciencias de la Educación. Centro de Investigación Educativa, Colegio García Flamenco: El Salvador.
Roig Vila, Rosabel; Mengual Andrés, Santiago; Sterrantino Asmussen, Claudia y Quinto Medrano, Patricia (2015). "Actitudes hacia los recursos tecnológicos en el aula de los futuros docentes". @tic: revista dilnnovacióeEducativa, 15, pp.12-19. DOI: doi:10.7203/attic.15.7220.

Salinas, Jesús (2004). Innovación docente y uso de las TIC en la enseñanza universitaria. Revista de Universidad y Sociedad del Conocimiento (RUSC), 1(1), 1-14.

Sein-Echaluce, María Luisa; Fidalgo-Blanco, Ángel y Alves, Gustavo (2016). "Technology behaviors in education innovation". Computers in Human Behavior, 72, pp.596-598. DOI: http://dx.doi.org/10.1016/j.chb.2016.11.049.

Sivan, Atara (2000). "The implementation of peer assessment: an action research approach". Assessment in Education, 7(2), pp.193-213. DOI: http://dx.doi.org/10.1080/713613328.

Torres Santomé, Jurjo (1996). Globalización e interdisciplinariedad: El currículum integrado. Madrid: Morata.

Zoppi, Ana María (1995). El pensamiento en la práctica de los docentes generadores de innovaciones curriculares. Segundo Informe Académico. San Salvador de Jujuy: Universidad Nacional de Jujuy. Secretaría de Ciencia, Técnica y Estudios Regionales.

| Cita recomendada de este artículo

Gómez Trigueros, Isabel María. (2017). “Los proyectos de innovación como recurso formativo en el Máster del Profesorado”. en @tic. revista d'innovació educativa. Número 19. Otoño (Julio-Diciembre 2017), pp. 22-29. 